\title{
Drug use trajectory patterns among older drug users
}

This article was published in the following Dove Press journal:

Substance Abuse and Rehabilitation

I3 May 201 I

Number of times this article has been viewed

\section{Miriam Boeri \\ Thor Whalen \\ Benjamin Tyndall \\ Ellen Ballard}

Kennesaw State University, Department of Sociology and Criminal Justice, Kennesaw GA, USA

Correspondence: Miriam Boeri

Kennesaw State University,

Department of Sociology and Criminal

Justice, 1000 Chastain Road MD \#2204,

Kennesaw, GA 30I44-559I USA

Tel +l 6787972069

Email mboeri@kennesaw.edu
Abstract: To better understand patterns of drug use trajectories over time, it is essential to have standard measures of change. Our goal here is to introduce measures we developed to quantify change in drug use behaviors. A secondary goal is to provide effective visualizations of these trajectories for applied use. We analyzed data from a sample of 92 older drug users (ages 45 to $65)$ to identify transition patterns in drug use trajectories across the life course. Data were collected for every year since birth using a mixed methods design. The community-drawn sample of active and former users were $40 \%$ female, $50 \%$ African American, and $60 \%$ reporting some college or greater. Their life histories provided retrospective longitudinal data on the diversity of paths taken throughout the life course and changes in drug use patterns that occurred over time. Bayesian analysis was used to model drug trajectories displayed by innovative computer graphics. The mathematical techniques and visualizations presented here provide the foundation for future models using Bayesian analysis. In this paper we introduce the concepts of transition counts, transition rates and relapse/remission rates, and we describe how these measures can help us better understand drug use trajectories. Depicted through these visual tools, measurements of discontinuous patterns provide a succinct view of individual drug use trajectories. The measures we use on drug use data will be further developed to incorporate contextual influences on the drug trajectory and build predictive models that inform rehabilitation efforts for drug users. Although the measures developed here were conceived to better examine drug use trajectories, the applications of these measures can be used with other longitudinal datasets.

Keywords: drug use, trajectory patterns, mixed methods, older adults

\section{Introduction}

In this paper we introduce and discuss several measures designed to evaluate the discontinuous patterns of drug use trajectories over the life course of older drug users. A trajectory is defined as "a pathway or line of development over the life span". ${ }^{(\text {p227) }}$ A better conceptualization of the discontinuities of drug use patterns across the life course is considered essential to understanding drug use trajectories and consequences over time. ${ }^{2}$ For example, analysts may want to detect patterns and trends in the use trajectory or compare the drug use trajectories across different drugs. Clinicians might like to identify patterns in an individual drug user's trajectory in order to develop a hypothesis regarding the influences on these patterns. Here we describe the development of the measures that gauge the discontinuous aspects of drug use trajectories. Often a classification stems from an underlying characteristic that can be measured and subsequently used to study the phenomena. It is this characteristic that we will attempt to capture in a measure of discontinuity. 
Much existing research aimed at analyzing drug use trajectories pinpoint groups of trajectories and the possible causal characteristics of these. ${ }^{3-7}$ Most of these differentiate trajectories on the basis of their growth characteristics, that is, the evolution of drug use frequency over time, usually assessed on the basis of types of drug use level growth patterns. ${ }^{8-10}$ We will introduce measures to assess trajectory heterogeneity on the basis of the discontinuity the trajectories exhibit. In contrast with growth models, here we are interested in significant changes of drug use in itself, not particularly if the change is part of a decreasing, persistent, or increasing trend, or belonging to a particular growth pattern. We use the term discontinuity defined as a descriptive change in manifest behavior over the life course. ${ }^{2}$

We propose that the measures we introduce here can be effectively used to gauge trajectory discontinuity and that identifying discontinuous patterns has important implications for future research and applications. This proposition is supported by earlier work on continuity ${ }^{11}$ as well as more recent literature on substance use trajectories finding that "the dialectics of continuity and discontinuity still represent the core of development" across the life course. ${ }^{2(p 430)}$ A significant change in drug use may sometimes reflect important turning points in a life course; therefore the discontinuity of drug use trajectories may be an informative indicator in itself. ${ }^{12}$ For example, incarceration might influence the cessation of certain illegal drugs, and re-entering society from prison might be related to using again. A more subtle transition in drug use might occur when a user begins a relationship with someone who does not use drugs, and is motivated to cease using. There are a myriad of influential factors on drug use trajectories, and having a measure of trajectory discontinuity that locates transition in use helps to identify contextual influence by exact periods of time. As suggested by others, challenges in existing trajectory analyses include distinguishing temporary from long-term transitions and deciding on the optimal time interval to capture sequences over time. ${ }^{2}$ Although the measures developed here were conceived with drug use in mind, the applications of these techniques are not restricted to drug use alone. These measures can be used with other longitudinal datasets with binary variables representing a trajectory. How they are used depends on the data characteristics. Here we describe the measures using the characteristics of drug use trajectories.

Our goal was to evaluate the discontinuous characteristics of trajectories and to develop measures that can be used to acquire insight and/or compare the discontinuity of drug use of certain users and/or typical use dynamics of certain drugs.
We gauged drug use on a 2-point scale: active (used at least once in a given year) and non-active (never used in a given year). Transitions from non-active to active, or from active to non-active, are notable events in such binary trajectories. It is the dynamics of these transitions that we aim to capture in the measures developed herein.

We begin by simply counting the number of transitions in a trajectory, which we call a transition count, and examine what basic properties this number expresses in the sense of what this particular measure tells us about the characteristics of the trajectory. Next, incorporating time since first use into the raw transition count, we define a measure of transition rate. These two measures are basic and are not, as is, good candidates for inferential extensions. On the other hand, they are useful tools to describe and diagnose drug use data, pinpoint outliers, detect patterns, and possibly percolate interesting hypotheses. Hypotheses are not included in this paper on the development of measures, but we discuss how they can be generated using these measures. Transition rates lead up to what we call relapse and remission rates, which measure distinct transitions from non-active to active use and from active use to non-active. These latter measures not only provide another perspective on the data, but are also more apt for the development of inferential statistics and, therefore, of generalizations and extensions.

\section{Methods}

The measures we describe in this paper were conceived as we attempted to analyze data we collected from older drug users. The goal of that research was to identify transitions in drug use trajectories and risk behaviors throughout the lives of the older adults. Existing measures of transitions did not fit our purposes. Here we describe the methods used in this study on older adult drug users to explain where and how the data employed to illustrate our new measures were derived. A sample of 92 older drug users provided the data analyzed to identify transition patterns in drug trajectories across the life course.

\section{Sample and data collection}

The data collection included both qualitative and quantitative methods and analysis using a retrospective longitudinal design. Data were collected between August 2009 and October 2010. To be eligible, respondents had to be at least 45 years old at the time of the interview and either active or former users of heroin, cocaine/crack or methamphetamine. These drugs were chosen because they represent the three major drugs of use associated with the most severe consequences. ${ }^{3,9}$ 
Polydrug use was the norm among our sample, as is found in some contemporary studies. ${ }^{13,14}$ Active use was defined as having used one of these drugs in the past year. Former use was defined as having used at least one of these drugs in the past ten years, for at least six consecutive months, but not used any of these drugs in the past year or longer. We chose ten years as the period within which cessation occurred in order to have both those who ceased use for many years as well as those who ceased use more recently. One year is chosen as the time period of no drug use since it incorporates the relapse period identified in a review of relapse literature. ${ }^{15}$ Transition in drug use from active and non-active was measured in one-year time periods, which we identified to be the smallest period of time with a reliable amount of recall accuracy over the entire life course.

The study sample was recruited from the metropolitan area of a large southeastern city in the USA. We employed a combination of ethnographic mapping, snowball and targeted sampling methods, including field observation, community consultants, fliers, and referrals. ${ }^{16,17}$ These methods have proven effective in recruiting respondents from hidden and hard-to-reach populations, such as noninstitutionalized drug users. ${ }^{18-20}$ Guided by theoretical sampling, ${ }^{21}$ the final sample is diverse in terms of age, race, and years of use. About half of our sample identified as active users. Ages range from 45 to 65 years old. About half of the sample is African American, four identified as "other" (American Indian or Latino), and the remainder white. A little over $40 \%$ of the sample is female. About a quarter have a high school education or its equivalency, with almost $60 \%$ reporting some college experience or more. For this paper, the sample characteristics are not important in the development of the transition and discontinuity measures other than its diversity in terms of drug use trajectory heterogeneity, which is why an older user sample with a long drug history was advantageous.

We developed an innovative combination of quantitative and qualitative strategies to collect life history interviews. The life history interview is a research tool designed to provide data with distinguishable transitions throughout the life course. ${ }^{22,23}$ The life histories of older adults provide rich retrospective longitudinal data on the diversity of paths taken throughout the life course and changes that occur over time. ${ }^{1,24-27}$ The data provided self-reported histories of drug use, risk behaviors and social contextual variables.

A well-developed body of research shows that the validity of self-reported data is increased when a number of proven methods are employed. ${ }^{28-32}$ For example, providing interviewers and respondents with a form of self-checking, ${ }^{33}$ establishing rapport, ${ }^{34}$ and using cues, such as a historical event timeline, helps ensure reliable data is collected on substance use and abuse. ${ }^{35}$ Challenges to the reliability and validity of longitudinal data include two forms of biases: recall accuracy and social desirability. ${ }^{36}$ While self-reports in cross-sectional and prospective longitudinal designs have fewer recall challenges than those collected in retrospective designs, they are influenced by social desirability biases that limit reporting of stigmatized or illegal behaviors. ${ }^{23,37}$ Retrospective data is less prone to this kind of social desirability effect and has the advantage of being cheaper to gather, as opposed to prospective longitudinal designs. ${ }^{38}$ Additionally, using a mixed methods design has been shown to be particularly important to avoid the problems associated with social desirability. ${ }^{37,39}$ Our retrospective longitudinal and mixed method design incorporated all of these strategies to provide reliable and valid data.

Potential respondents were screened to ensure compliance with the eligibility criteria. Interviews were conducted face-to-face in a private setting which would make the respondent feel at ease. Two well-trained interviewers were present with each respondent during the interview and continually cross-checked the data as it was collected. The entire interview process lasted between three and five hours. Respondents were offered food and given $\$ 40$ at the end of the interview. Reimbursement for respondents has been shown to be ethical and effective when collecting research on stigmatized behaviors such as drug use. ${ }^{40,41}$ The Institutional Review Board at the investigators' university approved the interview protocol.

Both quantitative and qualitative data were collected concurrently using a computer assisted personal interview (CAPI) and audio-recorded in-depth interview. Interviews began with the in-depth narrative interview wherein the respondents were free to discuss their life story with minimal guidance by the interviewer. Interviewers wrote important events, social roles, and behaviors on a paper life history matrix that allowed them to organize and keep track of time-specific facts and events in the life of the respondents. The matrix and notes informed and guided the interviewers as they collected the data on the computer survey. Inconsistencies and gaps in the respondent's stories could be quickly and easily caught and clarified by interviewers, enhancing the reliability of the survey data. Additionally, a timeline with important historical events was provided to the respondents to help give them memory cues and historical context in which to place events in their lives. For example, respondents were reminded 
that "this was the year Kennedy was shot" as data were collected for 1963. Diverse timelines were developed to be culturally sensitive.

\section{Variables}

The survey consisted of questions about residence, family, work, relationships, health, education, and criminal justice involvement and drug use for every year. Questions used for this paper regard yearly use and non-use of each of the following drugs: tobacco (TOB); marijuana (MAR); alcohol (ALC); hallucinogens (HAL); cocaine (COC); crack cocaine (CRK); heroin (HER); amphetamine (AMP); methamphetamine (MET) and prescription pill misuse (PRP). Prescription pills were reported as active use only when they were obtained illegally or when a prescription was being misused. Other drug use variables (eg, route of administration, addiction severity) were collected but not used in the analysis for this paper.

Drug use frequency, recorded on an 8-point scale, was re-coded to a 2-point scale so that drug use of a given user at a given age (year) is expressed by one of two values:

0: Non-active (never used drug $\mathrm{X}$ that year)

1: Active (used drug $\mathrm{X}$ at least once that year)

A binary measure has several advantages: 1) it enables a more parsimonious description and discussion of the measures we developed; 2) it attends to issues regarding the validity of self-reported data (ie, recall of use and non-use each year over many years is plausibly more valid than recall of frequency of use for every year over the life course); 3 ) the transitions between use and non-use are probably more noteworthy than any other transition between two drug use levels and probably reflect a major change in the user's drug career; 4) analyzing such binary trajectories complements the existing growth models perspective since the latter cannot really be applied to binary trajectories as they stand, without adapting the usual definitions and interpretations (for example, what would a "steadily increasing" drug use trajectory be if use were to only be captured by a binary variable?); 5) the difference between growth aspects and discontinuity aspects of trajectories is not so clear when looking at multiple use levels of drug use trajectories.

\section{Descriptive statistics for trajectory discontinuity}

In the following we describe how and why the measures and visualizations we used were developed, based on analysis of our study data. For the sake of brevity, we first use hypothetical examples to explain the foundational development and progression of the measures. We follow this with visualizations of actual users selected from our study sample that illustrate the measures and their potential applied use.

\section{Drug use trajectory}

The (binary) trajectory of a user for a specific drug is fully described by a sequence, $\mathrm{T}$, of $0 \mathrm{~s}$ and $1 \mathrm{~s}$ specifying the drug use of a respondent for each age of the life of a user (starting at age 0 and ending at the age the user was at the time of the interview). The left-most digit corresponds to 0 years old and digits are grouped by groups of five to facilitate readability. For example, consider the following hypothetical trajectory for tobacco:

$$
\mathrm{T}=000000000001111111111000000000111 \text {. }
$$

This trajectory describes a user who took tobacco for the first time at 11 years old. The user then continuously (meaning "at least once a year") used tobacco until 21 years old when she stopped, only to start again at 30 years of age and remain active until 32 years of age, at the time she was interviewed.

\section{Trajectory discontinuity}

The discontinuous patterns of all drug trajectories are visualized in the following graphs that include both drug use and selected risk behavior variables, based on two individual users drawn from our study. The images in Figure 1 exhibit the trajectories of two actual users for each of the 10 drugs in the survey. A row represents a trajectory for a given drug over the life of the individual. A black shaded cell for a given age (x-axis) and drug (y-axis) represents active use (1) and a non-shaded cell non-active (0).

As shown, both respondents depict long stretches of active use of tobacco and both have used a diversity of drugs in their lives. While respondent 113 has had longer periods of using heroin and crack that reveal a discontinuous pattern of active and non-active years, respondent 026 has a more discontinuous pattern of using prescription pills and drinking alcohol. Recall that prescription pills are included only when they are misused or obtained illegally. Although 113 reports many years of self-reported problematic drug use, as seen in the row labeled "problematic", alcohol does not appear to be a problematic substance in his life. We can easily see that this respondent has had over 20 years of continuous treatment and is non-active for all drugs in the past three years. Contrast this with respondent 026 who, although he is in treatment the year he was interviewed, has had few continuous years of treatment and has not stopped his illegal use of prescription drugs. 

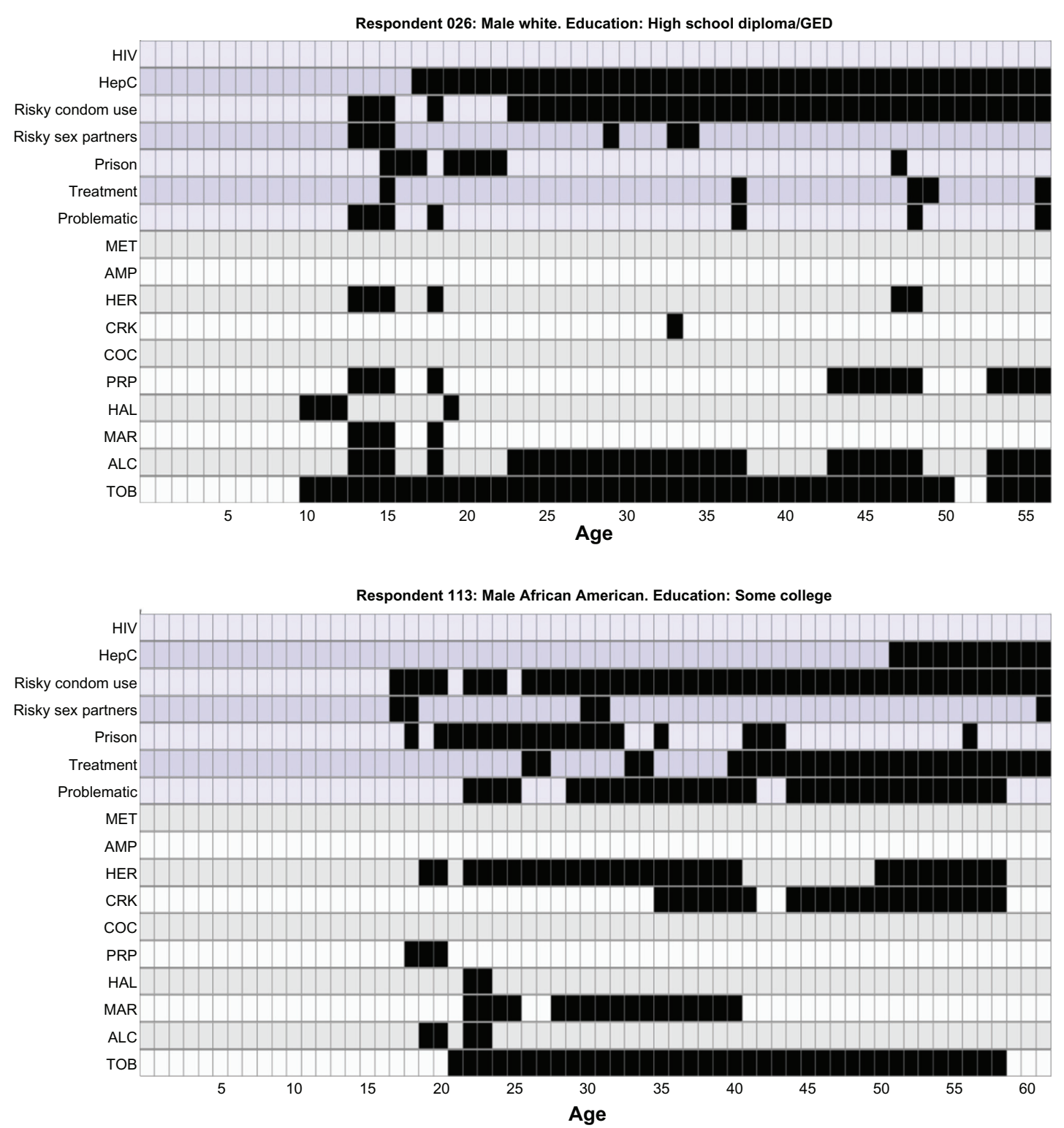

Figure I Drug use trajectories of all drugs and selected risk variables for two respondents.

Note: Black shaded cell represents active use.

Both respondents have hepatitis $\mathrm{C}$ and have spent time in prison or jail. The graphs present a parsimonious description for the variables of interest over an entire life course. Later in this paper, we return to these two respondents to show how measures of discontinuity can be used to estimate the probability that a user will remain active or non-active in the next year for specific drugs based on their trajectories.

The graphs are presented here to better visualize the measures of discontinuous trajectory patterns that we explain in the next section in more detail. The risk variables are shown here only to illustrate future applications of the model and are not further explored in this paper. We also do not address the numerous causal influences on transitions in drug use, since it is beyond the scope of this paper. As mentioned previously, many aspects of a life course will affect and be affected by drug use. For example, loss of employment or divorce may result in a relapse and conversely, acquiring a new job or spouse may influence cessation of use. We leave causal influences on drug use for a future application of the techniques we describe here. In this paper, our aim is to describe the development of measures that gauge the discontinuous aspects of drug use trajectories. Firstly we will introduce several descriptive statistics based on the number of transitions that appear in drug use trajectories. We will discuss what particular aspects of a trajectory these measures convey and illustrate this using the trajectory visualizations from our older drug user study. 


\section{Transition count}

We start with a straightforward measure that we will refer to as transition count (TC). The TC of a trajectory $\mathrm{T}$ is defined to be the number of sequential transitions from 0 (non-active) to 1 (active) or from 1 (active) to 0 (non-active). For example, consider the following hypothetical trajectory for alcohol:

$$
\mathrm{T}=0000000111100001111 .
$$

The transition count of $\mathrm{T}$ is $\mathrm{TC}(\mathrm{T})=3$. Indeed, our hypothetical user started (as all users do) with a sequence of $0 \mathrm{~s}$ and had his first alcoholic drink at age 7. This is the first transition (from non-active to active). The transition count for our hypothetical case is incremented by one. The user drank at least once a year until age 10, but did not drink when he was 11 years old. This is the second transition (from active to non-active), so the transition count is incremented again. Nothing changed until age 15 when the user drank again, and the transition count is incremented once more. Since our hypothetical user continued drinking until age 19, the age he was when he was interviewed, his transition count is 3 , shown by the three bolded numerals indicating a transition.

Transition count aspects are fundamental in the analysis of drug use trajectories. They provide a single descriptive statistic that captures several characteristics and can therefore be a useful statistic to diagnose the data, such as identifying outliers and uncovering biases. For example, the transition count encodes several noteworthy properties of trajectories: 1) if the TC is 0 , it means that the individual never used that particular drug; 2) a TC of 1 means that the individual started and never stopped the drug (at the level of year-by-year observations); 3) a TC of 2 means that the individual used the drug at some point, but then stopped indefinitely (that is, until the time of the interview); 4) an even TC means that the individual is NOT currently (at the time of the interview) using the drug; and 5) an odd TC means that the individual is currently using the particular drug.

Transition counts can also be used to compile descriptive tables to compare the dynamics of specific drugs. Table 1 displays the range of TCs (0-12) found in our sample of older drug users, as well as the frequency distribution of respondents that fell into each TC bracket for every drug for all 920 trajectories ( 92 respondents for 10 drugs). The range of TCs shows the large variation among users. For example, some respondents reported that they never used marijuana ( $\mathrm{TC}=0$ ), while one respondent reported transitioning 12 times before discontinuing marijuana. Table 2 shows the frequency distribution of trajectories as percentages for each TC count for every drug. Viewing the data in frequency tables allows us to compare the trajectories for each drug and identify interesting trends in our data. For example, Table 2 indicates that the majority of respondents (56.5\%) never quit using tobacco once they begin while the majority of respondents who had used hallucinogens (42.4\%) discontinued use and never took this drug again.

However, the transition count has some shortcomings in emulating how one might commonly compare the discontinuity of several trajectories. One critical inadequacy is that transition count alone does not take into account the amount of time the user has had to accrue these transitions, and this could be misleading. For example, consider the following two hypothetical trajectories in Figure 2.

If we base our judgment on the transition count, respondent $Y$ has a more discontinuous use pattern than respondent $X$. To make the matter of comparing the discontinuity of a trajectory more pertinent to the dynamics of a user once he/she is exposed to the given drug, we suggest it is more appropriate to only consider dynamics during the period since the first use of the drug. If we extrapolate the drug use of respondent $\mathrm{X}$ to the same span of use as that of respondent $Y$, then respondent $\mathrm{X}$ displays much more discontinuity. To capture this we need a measure evaluating the tendency of a user to transition rather than the number of transitions itself.

\section{Transition rate}

One straightforward way of integrating the duration of the respondent's career with a drug into a transition count is to divide the latter by the number of years since the year of first use. We thus define the transition rate (TR) of a trajectory only for trajectories with non-null transition counts (null meaning the participant never used this drug) as follows:

$\mathrm{TR}=(\mathrm{TC}-1) /$ number of years since first use.

The transition rate gives us a sense of how often transitions occur during the respondent's career with this drug.

In our hypothetical example above, respondent $\mathrm{X}$ has a TR of $(5-1) / 9=0.4444$ and respondent $Y$ has a TR of $(7-1) / 45=0.1333$, thereby conveying that respondent X's drug use is considerably more discontinuous than respondent Y's drug use. Transition rates are comprised between 0 (corresponding to a respondent that uses every year since the onset) and 1 (corresponding to a trajectory that toggles from active to non-active every year).

Transition rates let us see the duration of the career with the number of transitions for each individual. So for example, if someone has a TC of 4 but started 20 years ago and still uses, this is not such a discontinuous career as someone with a TC of 4 who started only 4 years ago. Unlike TCs, the TRs range over many unique values. To better compare these ranges, 
Table I TC frequency count

\begin{tabular}{|c|c|c|c|c|c|c|c|c|c|c|}
\hline & \multicolumn{10}{|c|}{ Drugs } \\
\hline & ТОВ & ALC & MAR & HAL & PRP & COC & CRK & HER & AMP & MET \\
\hline \multicolumn{11}{|c|}{ TC frequency count } \\
\hline 0 & 9 & 6 & 4 & 37 & 37 & 11 & 18 & 32 & 50 & 54 \\
\hline I & 52 & 22 & 12 & 1 & 6 & 6 & 17 & 8 & 1 & 3 \\
\hline 2 & 8 & 24 & 36 & 39 & 21 & 34 & 23 & 17 & 32 & 17 \\
\hline 3 & 15 & 11 & 13 & 0 & 4 & 4 & 9 & 5 & 0 & 8 \\
\hline 4 & 3 & 10 & 16 & 12 & 11 & 22 & 13 & 16 & 7 & 7 \\
\hline 5 & 3 & 7 & 2 & 0 & 6 & 3 & 6 & 3 & 0 & I \\
\hline 6 & 0 & 3 & 4 & 3 & 2 & 8 & 3 & 7 & 1 & 2 \\
\hline 7 & 2 & 5 & I & 0 & 3 & 0 & I & 2 & 0 & 0 \\
\hline 8 & 0 & I & I & 0 & 2 & 3 & I & 2 & 0 & 0 \\
\hline 9 & 0 & 1 & I & 0 & 0 & 0 & I & 0 & 0 & 0 \\
\hline 10 & 0 & I & I & 0 & 0 & I & 0 & 0 & I & 0 \\
\hline II & 0 & 1 & 0 & 0 & 0 & 0 & 0 & 0 & 0 & 0 \\
\hline 12 & 0 & 0 & I & 0 & 0 & 0 & 0 & 0 & 0 & 0 \\
\hline
\end{tabular}

Abbreviations: TOB, tobacco; ALC, alcohol; MAR, marijuana; HAL, hallucinogens; PRP, prescription pill misuse; COC, cocaine; CRK, crack cocaine; HER, heroin; AMP, amphetamine; MET, methamphetamine; TC, transition count.

Table 2 TC frequency distribution

\begin{tabular}{|c|c|c|c|c|c|c|c|c|c|c|}
\hline & \multicolumn{10}{|c|}{ Drugs } \\
\hline & ТОВ & ALC & MAR & HAL & PRP & COC & CRK & HER & AMP & MET \\
\hline \multicolumn{11}{|c|}{ TC frequency distribution (in \%) } \\
\hline 0 & 9.8 & 6.5 & 4.3 & 40.2 & 40.2 & 12.0 & 19.6 & 34.8 & 54.4 & 58.7 \\
\hline I & 56.5 & 23.9 & 13.0 & 1.1 & 6.5 & 6.5 & 18.5 & 8.7 & 1.1 & 3.3 \\
\hline 2 & 8.7 & 26.1 & 39.1 & 42.4 & 22.8 & 37.0 & 25.0 & 18.5 & 34.8 & 18.5 \\
\hline 3 & 16.3 & 12.0 & 14.1 & 0.0 & 4.3 & 4.3 & 9.8 & 5.4 & 0.0 & 8.7 \\
\hline 4 & 3.3 & 10.9 & 17.4 & 13.0 & 12.0 & 23.9 & 14.1 & 17.4 & 7.6 & 7.6 \\
\hline 5 & 3.3 & 7.6 & 2.2 & 0.0 & 6.5 & 3.3 & 6.5 & 3.3 & 0.0 & I.I \\
\hline 6 & 0.0 & 3.3 & 4.3 & 3.3 & 2.2 & 8.7 & 3.3 & 7.6 & I.I & 2.2 \\
\hline 7 & 2.2 & 5.4 & I.I & 0.0 & 3.3 & 0.0 & I.I & 2.2 & 0.0 & 0.0 \\
\hline 8 & 0.0 & 1.1 & I.I & 0.0 & 2.2 & 3.3 & I.I & 2.2 & 0.0 & 0.0 \\
\hline 9 & 0.0 & 1.1 & I.I & 0.0 & 0.0 & 0.0 & I.I & 0.0 & 0.0 & 0.0 \\
\hline 10 & 0.0 & I.I & I.I & 0.0 & 0.0 & I.I & 0.0 & 0.0 & I.I & 0.0 \\
\hline II & 0.0 & I.I & 0.0 & 0.0 & 0.0 & 0.0 & 0.0 & 0.0 & 0.0 & 0.0 \\
\hline 12 & 0.0 & 0.0 & I.I & 0.0 & 0.0 & 0.0 & 0.0 & 0.0 & 0.0 & 0.0 \\
\hline
\end{tabular}

Abbreviations: TOB, tobacco; ALC, alcohol; MAR, marijuana; HAL, hallucinogens; PRP, prescription pill misuse; COC, cocaine; CRK, crack cocaine; HER, heroin; AMP, amphetamine; MET, methamphetamine; TC, transition count.
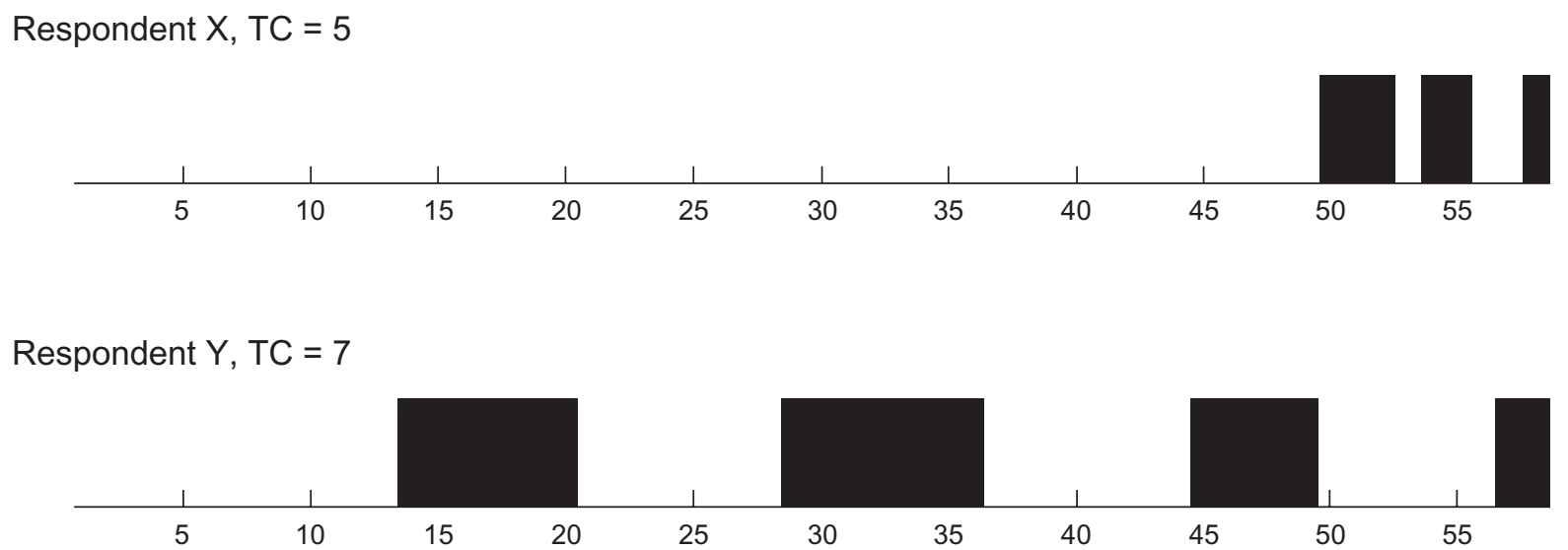

Figure 2 Transition count trajectories.

Notes: Line represents age trajectory in years. Black shaded area represents active use for given years

Abbreviation: TC, transition count. 
we computed the frequencies over 11 defined categories (brackets), which encompass a percentile range of values. To compute these values we performed a number of statistical calculations including: 1) normalizing the TRs within a same drug using Z-scores; 2) taking the mean of these z-scores to describe an average discontinuity of a respondent over all drugs; 3) taking the z-score of this mean to see where this general discontinuity lies compared to other respondents.

Tables 3 and 4 show the bracketed TRs calculated from the z-score of the mean of the z-scores of the TRs, as described above. These cumulative frequency percentages help us to compare the distribution of the TRs for one drug with the general TR distribution for all drugs. The first bracket contains those TRs equal to 0 (started and never stopped use of the drug), since a good number of trajectories fall under this category. The subsequent 10 brackets are defined by the deciles of the TRs for all respondents and all drugs. These deciles are 0.0250, 0.0286, $0.0323,0.0435,0.0625,0.0769,0.0932,0.1197,0.1613$.

Table 3 shows the range of TRs found in our sample, as well as the frequency count of respondents that fell into each TR bracket for every drug. Unlike the 0 in the TC, a TR equal to 0 means that, at the time of the interview, the respondent has continued to use a particular drug every year since initiating use and never stopped. Table 4 displays the frequency distribution of trajectories as percentages for each TR bracket for every drug. As with the TC tables (Tables 1 and 2), these tabular representations help us compare the TRs for each drug to clarify trends in our sample.

We include these tables to illustrate how the measures and statistics depicted can be used to generate hypotheses regarding trends, patterns, and comparisons between the trajectories of different drugs. For example, Table 4 shows that $27 \%$ of CRK users or former users fall in the last "TR greater or equal to 0.1613 " bracket. This is the highest percent in this bracket for all drugs. This value of 0.1613 is the 90 th percentile of TRs over all drugs, meaning that $90 \%$ of all defined TRs are smaller than this value. The fact that $27 \%$ of CRK users have this transition rate level speaks again of the highly discontinuous behavior of CRK use, compared to the behavior with other drugs in our sample. Further examination of these tables is beyond the scope of this paper. Instead we move to the next step in our development of measures to gauge trajectory discontinuity.

The transition rate exhibits the tendency to transition from one drug use state to another, but it does not indicate whether the transitions are from active use to non-active or vice versa. Consider the following hypothetical trajectories shown in Figure 3.

\section{Remission rate and relapse rate}

Respondents $\mathrm{V}$ and $\mathrm{W}$ both have same transition rate and transition count, yet there is a noteworthy difference between them. Respondent $\mathrm{V}$ was active most of his life, with the exception of a few short remissions into non-use; whereas, by contrast, respondent $\mathrm{W}$ was non-active most of her life with the exception of a few short relapses into use. The transition count and rate aggregate remissions and relapses, without taking into account the respective durations of the periods they initiate.

The transition rate is in fact an estimate of the underlying probability to transition from one state to another. We will define the remission and relapse rates so as to be estimates of the underlying probability to transition from non-active to active and from active to non-active, respectively. This

Table 3 TR frequency count of respondents in each percentile bracket

\begin{tabular}{|c|c|c|c|c|c|c|c|c|c|c|}
\hline \multirow[t]{3}{*}{ Sample size } & \multicolumn{10}{|c|}{ Drugs } \\
\hline & ТОВ & ALC & MAR & HAL & PRP & COC & CRK & HER & AMP & MET \\
\hline & 83 & 86 & 88 & 55 & 55 & 81 & 74 & 60 & 42 & 38 \\
\hline \multicolumn{11}{|l|}{ TR frequency count } \\
\hline $\mathrm{TR}=0$ & 52 & 22 & 12 & 1 & 6 & 6 & 17 & 8 & 1 & 3 \\
\hline $0.0000<\mathrm{TR}<0.0250$ & 4 & 9 & 9 & 6 & 2 & 4 & 0 & I & 6 & 0 \\
\hline $0.0250<=$ TR $<0.0286$ & 2 & 3 & 11 & 16 & 4 & 9 & 0 & 4 & 13 & 3 \\
\hline $0.0286<=\mathrm{TR}<0.0323$ & 0 & 9 & 12 & 8 & 8 & 2 & 0 & 2 & 5 & 2 \\
\hline $0.0323<=\mathrm{TR}<0.0435$ & 2 & 3 & 4 & 8 & 2 & 18 & 9 & 3 & 8 & 0 \\
\hline $0.0435<=\mathrm{TR}<0.0625$ & 13 & 9 & 11 & 1 & 3 & 2 & 3 & 5 & 0 & 8 \\
\hline $0.0625<=\mathrm{TR}<0.0769$ & 1 & 5 & 10 & 4 & 4 & 4 & 5 & 4 & 2 & 5 \\
\hline $0.0769<=\mathrm{TR}<0.0932$ & 4 & 6 & 5 & 8 & 8 & 11 & 5 & 10 & 4 & 3 \\
\hline $0.0932<=\mathrm{TR}<0.1197$ & 3 & 6 & 4 & 1 & 9 & 10 & 5 & 8 & 1 & 6 \\
\hline $0.1197<=\mathrm{TR}<0.1613$ & 2 & 9 & 5 & 2 & 4 & 8 & 10 & 9 & 1 & 3 \\
\hline $0.1613<=\mathrm{TR}$ & 0 & 5 & 5 & 0 & 5 & 7 & 20 & 6 & 1 & 5 \\
\hline
\end{tabular}

Abbreviations: TOB, tobacco; ALC, alcohol; MAR, marijuana; HAL, hallucinogens; PRP, prescription pill misuse; COC, cocaine; CRK, crack cocaine; HER, heroin; AMP, amphetamine; MET, methamphetamine; TR, transition rate. 
Table 4 TR frequency percentage in each percentile bracket

\begin{tabular}{|c|c|c|c|c|c|c|c|c|c|c|}
\hline \multirow[t]{3}{*}{ Sample size } & \multicolumn{10}{|c|}{ Drugs } \\
\hline & ТОВ & ALC & MAR & HAL & PRP & COC & CRK & HER & AMP & MET \\
\hline & 83 & 86 & 88 & 55 & 55 & 81 & 74 & 60 & 42 & 38 \\
\hline \multicolumn{11}{|c|}{ TR frequency percentage } \\
\hline $\mathrm{TR}=0$ & 62.7 & 25.6 & 13.6 & 1.8 & 10.9 & 7.4 & 23.0 & 13.3 & 2.4 & 7.9 \\
\hline $0.0000<\mathrm{TR}<0.0250$ & 4.8 & 10.5 & 10.2 & 10.9 & 3.6 & 4.9 & 0.0 & 1.7 & 14.3 & 0.0 \\
\hline $0.0250<=\mathrm{TR}<0.0286$ & 2.4 & 3.5 & 12.5 & 29.1 & 7.3 & $\mathrm{II} . \mathrm{I}$ & 0.0 & 6.7 & 31.0 & 7.9 \\
\hline $0.0286<=\mathrm{TR}<0.0323$ & 0.0 & 10.5 & 13.6 & 14.6 & 14.6 & 2.5 & 0.0 & 3.3 & 11.9 & 5.3 \\
\hline $0.0323<=\mathrm{TR}<0.0435$ & 2.4 & 3.5 & 4.5 & 14.6 & 3.6 & 22.2 & 12.2 & 5.0 & 19.1 & 0.0 \\
\hline $0.0435<=\mathrm{TR}<0.0625$ & 15.7 & 10.5 & 12.5 & 1.8 & 5.5 & 2.5 & 4.1 & 8.3 & 0.0 & 21.1 \\
\hline $0.0625<=\mathrm{TR}<0.0769$ & 1.2 & 5.8 & 11.4 & 7.3 & 7.3 & 4.9 & 6.8 & 6.7 & 4.8 & 13.2 \\
\hline $0.0769<=\mathrm{TR}<0.0932$ & 4.8 & 7.0 & 5.7 & 14.6 & 14.6 & 13.6 & 6.8 & 16.7 & 9.5 & 7.9 \\
\hline $0.0932<=\mathrm{TR}<0.1197$ & 3.6 & 7.0 & 4.5 & 1.8 & 16.4 & 12.4 & 6.8 & 13.3 & 2.4 & 15.8 \\
\hline $0.1197<=\mathrm{TR}<0.1613$ & 2.4 & 10.5 & 5.7 & 3.6 & 7.3 & 9.9 & 13.5 & 15.0 & 2.4 & 7.9 \\
\hline $0.1613<=\mathrm{TR}$ & 0.0 & 5.8 & 5.7 & 0.0 & 9.1 & 8.6 & 27.0 & 10.0 & 2.4 & 13.2 \\
\hline
\end{tabular}

Abbreviations: TOB, tobacco; ALC, alcohol; MAR, marijuana; HAL, hallucinogens; PRP, prescription pill misuse; COC, cocaine; CRK, crack cocaine; HER, heroin; AMP, amphetamine; MET, methamphetamine; TR, transition rate.

type of model of a sequence of states is typically known as a "Markov Model" or "Markov Chain" and is defined as a sequence of states having the property that the next state depends only on the current state.

A Markov Chain specifies the probability of the next state of the sequence conditional on the current state. In our case we have two states ( 0 and 1$)$, so four transition probabilities need to be specified, namely $\operatorname{Prob}(0 \rightarrow 0), \operatorname{Prob}(0 \rightarrow 1)$, $\operatorname{Prob}(1 \rightarrow 0)$, and Prob $(1 \rightarrow 1)$. In Markov Chain terminology, not only $0 \rightarrow 1$ and $1 \rightarrow 0$, but also $0 \rightarrow 0$ and $1 \rightarrow 1$ are called "transitions". That is, respondents can take 1 of 4 states in any given year: no transition from nonuse, transitioning from nonuse to use, transition from use to nonuse, and no transition from use. Let us take the trajectory of respondent $\mathrm{V}$ as an example. As in the case of the transition rate discussed above, we will only consider the sub-trajectory corresponding to the interval between the onset of drug use (first year that this drug was used) and the year of the interview. In the case of respondent $\mathrm{V}$, we consider therefore the following trajectory:

\section{0.}

To estimate the probabilities of each possible type of transition, we count the number of $0 \mathrm{~s}$ and $1 \mathrm{~s}$ and the number of sequential pairs $00,01,10$, and 11 :

- $\quad \# 0$ : Number of $0 \mathrm{~s}=8$

- $\quad \# 00$ : Number of $00 \mathrm{~s}=6$

- $\quad \# 01$ : Number of $01 \mathrm{~s}=2$

- \#1: Number of $1 \mathrm{~s}=26$

- \#10: Number of $10 \mathrm{~s}=3$

- \#11: Number of $11 \mathrm{~s}=23$

From this information, we can estimate the probabilities of every possible sequential pair:

- $\operatorname{Prob}(0 \rightarrow 0) \sim \# 00 / \# 0=\# 00 /(\# 00+\# 01)=6 /(6+2)=0.75$

- $\operatorname{Prob}(0 \rightarrow 1) \sim \# 01 / \# 0=\# 01 /(\# 00+\# 01)=2 /(6+2)=0.25$

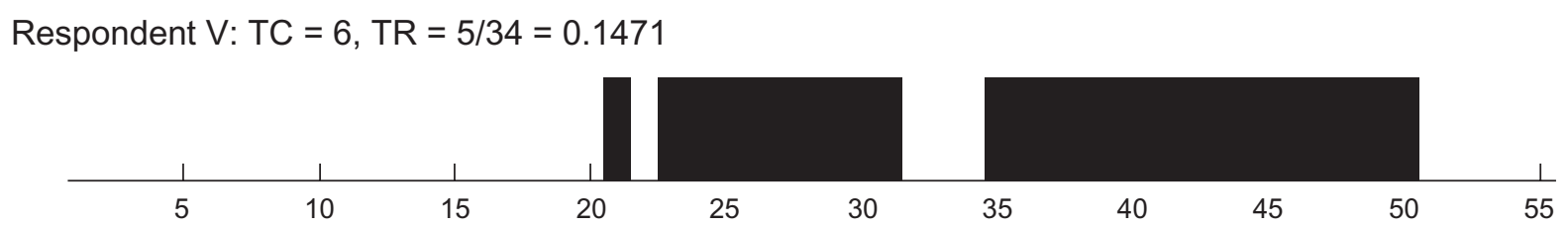

Respondent $\mathrm{W}: \mathrm{TC}=6, \mathrm{TR}=5 / 34=0.1471$

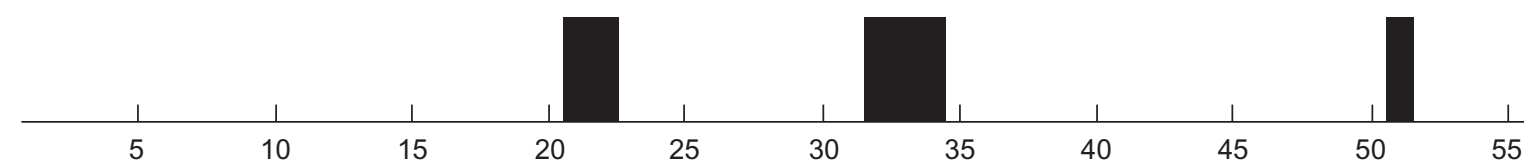

Figure 3 Transition rate trajectories.

Notes: Line represents age trajectory in years. Black shaded area represents active use for given years.

Abbreviations: TC, transition count; TR, transition rate. 
- $\operatorname{Prob}(1 \rightarrow 0) \sim \# 10 / \# 1=\# 10 /(\# 10+\# 11)=3 /(3+23)=0.12$

- $\operatorname{Prob}(1 \rightarrow 1) \sim \# 11 / \# 1=\# 11 /(\# 10+\# 11)=23 /$ $(3+23)=0.88$

This transition probability specification is commonly represented as a directed graph or "digraph" ${ }^{42}$ The directed graphs and transition matrices shown in Figure 4 were generated for hypothetical respondents $\mathrm{V}$ and $\mathrm{W}$. Once again, 1 represents active use in a given year and 0 non-active use of the drug in a given year. The percentages next to the directional arrows indicate the likelihood of remaining active (or non-active) in the next year or transitioning from active to non-active and vice-versa.

We see that respondent $\mathrm{V}$ tends to remain a user from year to year, with only a $12 \%$ chance of having a remission. Once a non-user again, he has a $75 \%$ chance of remaining so every year, but a non-negligible $25 \%$ chance of relapsing into use. In contrast, respondent $\mathrm{W}$, when in an active use year, has a $50 \%$ chance of a remission the next year. Once a non-user again, she tends to remain so, only relapsing into use with a $7 \%$ chance.

Note that the $0 \rightarrow 0$ and $1 \rightarrow 1$ can be deduced from the $0 \rightarrow 1$ and $1 \rightarrow 0$ probabilities, respectively. Indeed, these are inverse probabilities of each other. Hence the Markov chain transition probabilities are fully specified from the $0 \rightarrow 1$ and $1 \rightarrow 0$ transition probabilities only. These rates are calculated only from the first year of use onward. This brings us to the following definitions:

The relapse rate is the ratio of non-using years that were followed by a relapse into a using year. That is, the relapse rate is the number of $01 \mathrm{~s}$ divided by the number of $0 \mathrm{~s}$ (or equivalently, the sum of $01 \mathrm{~s}$ and the $00 \mathrm{~s}$ ).

The remission rate is the ratio of using years that were followed by a remission into a non-using year. That is, the relapse rate is the number of $10 \mathrm{~s}$ divided by the number of $1 \mathrm{~s}$ (or equivalently, the sum of $11 \mathrm{~s}$ and the $10 \mathrm{~s}$ ).

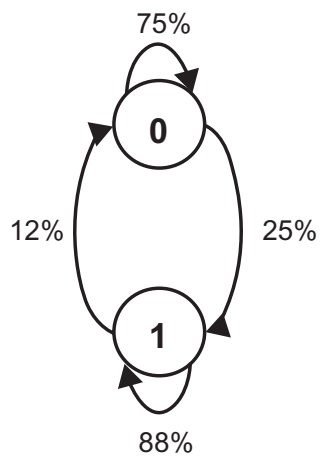

Respondent $\mathrm{V}$

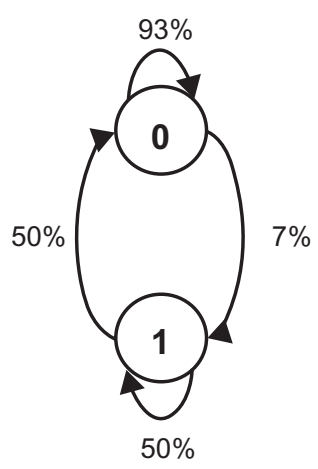

Respondent W
Figure 4 Directed graphs.

Notes: 0 represents non-active use in a given year. I represents active use in a given year.
Technically, relapse and remission rates can be seen as estimating the corresponding probabilities of an underlying Markov Chain model. In this model, each state is given a probability to be in that state the following year, given the state of the current year. Since drug use is measured here with a binary variable, the model is fully specified by two probabilities: the relapse probability (that the non-using respondent will use the following year) and the remission probability (that the using respondent will not use the following year). The probability that the non-using respondent will remain non-active the following year is one minus the relapse probability. The probability that the using respondent will remain active the following year is one minus the remission probability. To specify a Markov Chain completely, one should also specify a probability for the initial state of the sequence. Since we are considering trajectories and computing our rates only from the first year of use onward, our initial state will always be 1 (active user).

We combine both the transition count trajectory with the Markov Chain model depicted in the diagraph into one graph to provide a more complete view of the user's drug trajectory and probability for remission and relapse. Figures $5-8$ illustrate the drug trajectories and digraphs for the two actual respondents we discussed previously. We use alcohol and heroin trajectories for the purpose of comparison.

Comparing the trajectories and digraphs of two respondents' life use of alcohol and heroin, we see the trajectories reveal different patterns of discontinuity for each drug. Recall that the trajectory represents every year of life. The black shaded cells indicate active use that year (at least once) and the non-shaded cells indicate non-active. In this

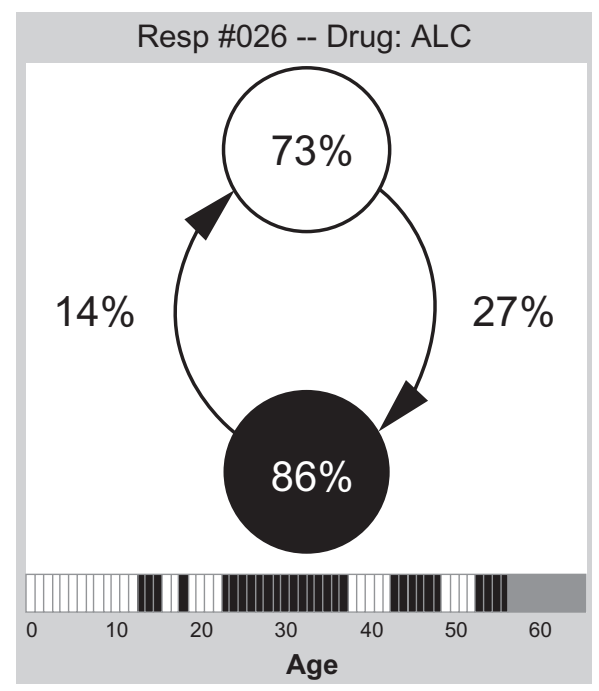

Figure 5

Notes: Trajectory line represents every year of life. Black shaded cell represents active use in a given year.

Abbreviation: ALC, alcohol. 


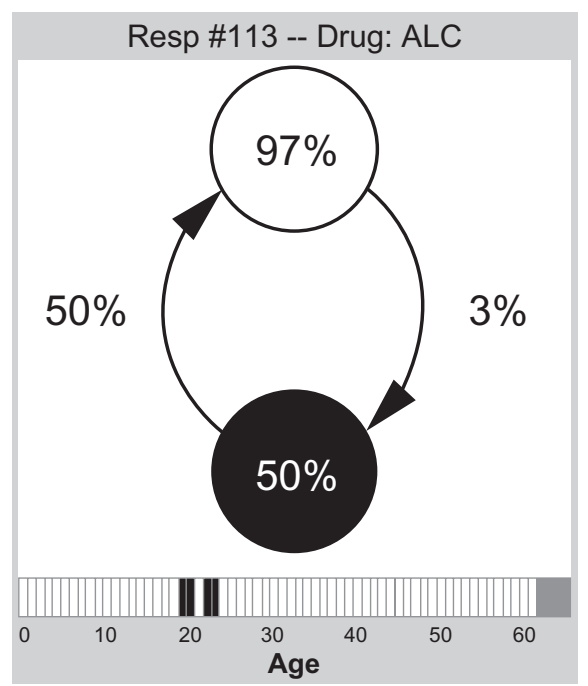

Figure 6

Notes: Trajectory line represents every year of life. Black shaded cell represents active use in a given year.

Abbreviation: ALC, alcohol.

graph, the grey shaded area starts the year after the age the respondent was interviewed: 026 was interviewed at age 56 and 113 at age 61 . The digraphs based on their trajectories show quite different probabilities for remission and relapse depending on the drug.

First we see that the respondents started alcohol at different ages (026 at age 13 and 113 at age 19). However, the trajectory of 026 shows a more discontinuous pattern, while 113 shows only a few years of active drinking around his twenties. Moreover, 113 has not had an alcoholic drink for almost 40 years, while 026 is drinking in the year of the interview. The digraphs reveal that 113 has a $97 \%$ probability to remain non-active in regards to alcohol. On the other

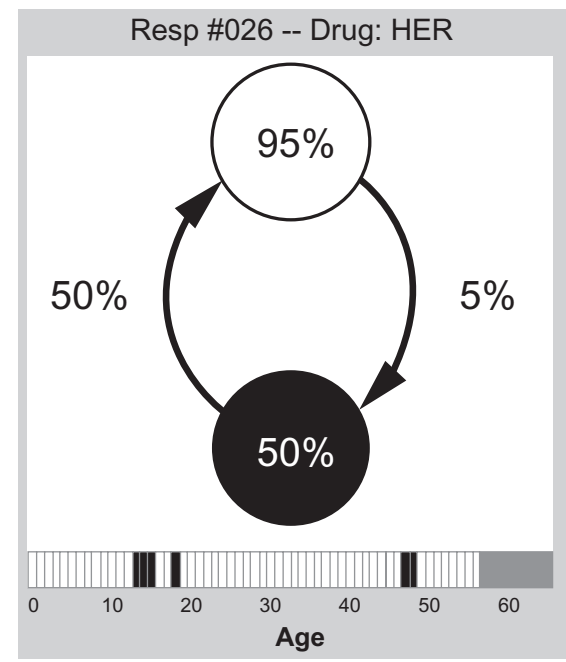

Figure 7

Abbreviation: HER, heroin.

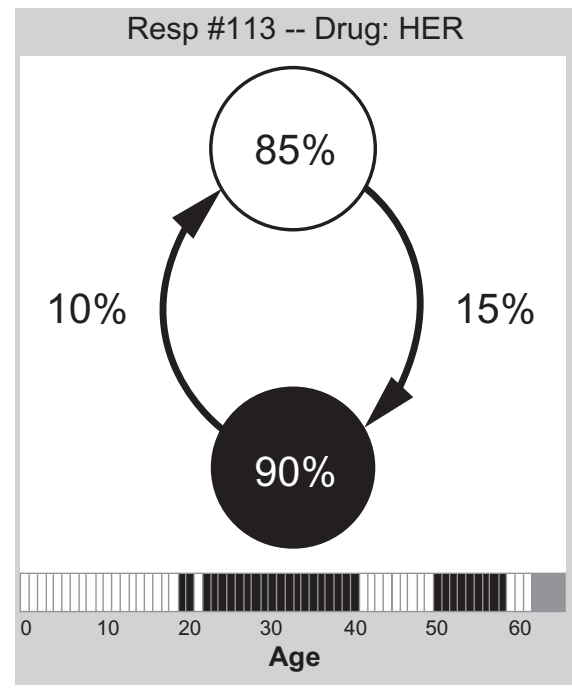

Figure 8

Notes: Trajectory line represents every year of life. Black shaded cell represents active use in a given year.

Abbreviation: HER, heroin.

hand, 026 is an active drinker in the year he was interviewed and, with a more discontinuous pattern of drinking and long periods of active use, he has an $86 \%$ probability of remaining active next year. These probabilities are almost completely reversed when looking at their heroin trajectories.

While both respondents show discontinuous patterns of heroin use, they are quite different. We see that 026 started using heroin at age 13 , and he stopped and started again in a few years' span before remaining non-active for almost 30 years. He was active for two years in his late forties and them remained non-active until the time of the interview. Instead, 113 started heroin later in life, at age 19, used for a few years, stopped one year and then remained active for almost twenty years. He stopped using heroin for another 9 years followed by a period of continuous active use for a 9-year span. While both are non-active at the time they were interviewed, the digraphs show that 113 has a $15 \%$ probability of relapsing the next year while 026 has only a $5 \%$ probability.

Space does not permit us to present the trajectory and digraph for all drugs used by 026 and 113, and a fuller description is beyond the scope of this paper. Yet, the trajectories for all drugs can be seen in the earlier graphs shown in Figure 1, and a brief discussion on this provides insight on the usefulness of these measures. Taking into consideration the drugs used each year shown in the graph for 026, he appears to have switched from using heroin to prescription pills. The qualitative interview (not presented here) revealed these are pain pills, which can act as a substitute for heroin. In addition, he has not stopped his drinking. By some treatment standards, 026 would not be viewed as rehabilitated or 
"in recovery". By contrast, 113 ceased all drugs and alcohol use for the last three years. Most treatment providers would consider 113 rehabilitated or at least in long term recovery. However, as shown above in the heroin digraph, while 113 has a considerable probability to remain in remission, he also has a greater probability for relapse than does 026 for heroin use. This outcome is the result of taking into account the discontinuity measures found in their life trajectories.

Based on our measures of transition counts and transition rates, the relapse and remission rates we compute from a trajectory can be used as estimates of the underlying relapse and remission probabilities of a given user for a given drug. Our objective in this article to develop measures that gauge the discontinuous patterns of a drug use trajectory are subsumed in these simple discontinuity statistics. Moreover our secondary goal to develop graphic models that describe the data visually is achieved through the trajectory graphs and digraphs presented here.

\section{Conclusion}

In this paper we responded to a number of methodological challenges to the identification of discontinuity in drug use trajectories. ${ }^{2}$ Framed in a life course perspective that includes both intra-individual change and inter-individual differences in trajectories across the life course, we distinguished temporary perturbations from long-term change. ${ }^{1}$ Using personperiod data from life history interviews with older drug users, we suggested the "year" as an optimal time interval. Furthermore, to better assess a trajectory, we proposed to collect yearly data from birth. We identified discontinuity in the yearly trajectory of drug use by analyzing the transitions from active to non-active (and vice-versa). Counting the total number of transitions, as measured by the transition count, was shown to be a useful subsuming measure of several fundamental types of trajectories. Incorporating the number of years since drug use onset, the transition rate gives us a more appropriate measure of discontinuity since it provides a sense of how often a user transitions. The relapse and remission rates take this further by exhibiting the contributions of transition from non-active to active use, and active to non-active, respectively. The new measures and statistics depicted in these tables and graphs can be used to identify trends and patterns, and compare the trajectories of different drugs or different groups of drug users. They provide a succinct way to model large event-history data sets that make it easier for researchers and practitioners to generate hypotheses and further their exploration on respective research questions.
In this paper, we developed measures to assess the discontinuity of drug use trajectories; however the measures can be employed with any sequence of binary variables. Here we applied them to drug use data in order to describe the development of the measures and to illustrate how they can be used. The developments of these measures and illustrative graphs have a number of potential applications for drug research and treatment. For example, the discontinuity measures presented here respond to the call for different approaches to address risk behaviors and the risk environment of drug users. ${ }^{43,44}$ By expanding on the measures shown here, we can develop inferential statistics to assess the risk of relapse and remission. Inferential statistics will also allow us to make better estimates of the underlying probabilities involved, thereby generating better estimates of the underlying activity and discontinuity of any risk behavior trajectory for which we have valid data.

\section{Limitations}

The assertions made here about the characteristics of the users and the drugs are purely descriptive of the sample, being only intended to articulate certain aspects that our measures exhibited. Used alone, these measures do not illustrate the whole picture. However, they can be used for comparing similarities and differences in the drug user trajectory across different drugs and for individual drug users. These measures and graphic visualizations provide a useful tool to gauge trajectory discontinuity. Hypotheses can be generated from the visualizations shown here, but validating such hypotheses will require developing inferential statistics based on these descriptive statistics. Inferential statistics provide tools to extend beyond the data itself and assess the validity of assertions about a more general context. We provided some indications on how such inferential statistics may be developed, but the development and discussion of these inferential statistics and their application will be left to future articles.

\section{Future research and applied use}

In this methodological paper we proposed new measures that provide tools to analyze discontinuous trajectories and support hypotheses generated by the graphic illustrations of these measures applied to data. Measuring and conceptualizing discontinuous patterns in individualized drug use has important implications for future research on drug use trajectories and potentially for predicting turning points in risk behaviors. Aggregated transition counts and transition rates may be used to compare trends in drug use patterns, across specific drugs, and across the life course of individual 
users. In addition, the visualization models presented here provide a parsimonious understanding of an individual user's life history of drug use and its association with selected variables. These measures can be modified and extended to predict risk of relapse, measured with the precision to individual differences and specific socio-contextual associations. Drug rehabilitation practitioners have identified a gap in research for individualized treatment approaches. ${ }^{45,46}$ The relapse and remission rates provide predictive value based on the user's life history and individualized trajectory, visually depicted in the digraphs. Our development of trajectory discontinuity measures is not finished. In future papers we will explore many more aspects of the trajectory, differences between types of drug users, active and inactive users, and other characteristics of the sample, as well as inferential statistical applications that incorporate the influence of social-contextual events over the life course. We envision eventual application of these models for drug treatment and rehabilitation purposes, as well as the development of healthcare intervention, prevention programs, and policy.

\section{Acknowledgment}

The project described was supported by Award Number 1R21DA025298-01A1 from the National Institute on Drug Abuse. The content is solely the responsibility of the authors and does not necessarily represent the official views of the National Institute on Drug Abuse or the National Institutes of Health. The authors would like to thank Craig Rafuse and David Gibson for their exceptional interviewing, the anonymous reviewers for their careful reading and insightful comments, and the older adults who shared their life histories with us.

\section{Disclosure}

The authors report no conflict of interests in this work.

\section{References}

1. Hser YI, Longshore D, Anglin MD. The life course perspective on drug use. Eval Rev. 2007;31(6):515-547.

2. Schulenberg JE, Maggs JL, O'Malley PM. How and why the understanding of developmental continuity and discontinuity is important. In: Mortimer JT, Shanahan MJ, editors. Handbook of the Life Course. New York: Plenum Publishers; 2003:413-436.

3. Brecht ML, Huang D, Evans E, Hser YI. Polydrug use and implications for longitudinal research: ten-year trajectories for heroin, cocaine, and methamphetamine users. Drug Alcohol Depend. 2008;96(3): 193-201.

4. Chassin L, Fora DB, King KM. Trajectories of alcohol and drug use and dependence from adolescence to adulthood: the effects of familial alcoholism and personality. J Abnorm Psychol. 2004;113(4):483-498.

5. Laub J, Sampson R. Integrating quantitative and qualitative data. In: Giele JZ, Elder GH Jr, editors. Methods of Life Course Research: Qualitative and Quantitative Approaches. Thousand Oaks (CA): Sage; 1998:213-229.
6. White HR, Pandina RJ, Chen PH. Developmental trajectories of cigarette use from early adolescence into young adulthood. Drug Alcohol Depend. 2002;65(2):167-178.

7. Yamaguchi K. Four useful finite mixture models for regression analyses of panel data with a categorical dependent variable. Sociol Methodol. 2008;38(1):283-328.

8. Ellickson PL, Martino SC, Collins RL. Marijuana use from adolescence to young adulthood: multiple developmental trajectories and their associated outcomes. Health Psychol. 2004;23(3):299-307.

9. Hser Y, Huang D, Brecht M, Li L, Evans E. Contrasting trajectories of heroin, cocaine and methamphetamine use. J Addict Dis. 2008;27(3):13-21

10. Juon H, Ensminger ME, Sydnor KD. A longitudinal study of developmental trajectories to young adult cigarette smoking. Drug Alcohol Depend. 2002;66(3):303-314.

11. Werner $\mathrm{H}$. The concept of development from a comparative and organismic point of view. In: Harris DB, editor. The Concept of Development: An Issue in the Study of Human Behavior. Minneapolis: University of Minneapolis Press; 1957:125-148.

12. Teruya $\mathrm{C}$, Yu F. Turning points in the life course: current findings and future directions in drug use research. Curr Drug Abuse Rev. 2010;3(3): 189-195.

13. Boeri MW, Sterk CE, Bahora M, Elifson KW. Poly-drug use among ecstasy users: separate, synergistic, and indiscriminate patterns. J Drug Issues. 2008;38:517-542.

14. Halkitis PN, Mukherjee PP, Palamar JJ. Multi-level modeling to explain methamphetamine use among gay and bisexual men. Addiction. 2007;102 Suppl 1:76-83.

15. Barrick C, Connors GJ. Relapse prevention and maintaining abstinence in older adults with alcohol-use disorders. Drugs Aging. 2002;19(8): 583-94.

16. Biernacki P, Waldorf $D$. Snowball sampling: problems and techniques of chain referral sampling. Sociol Methods Res. 1981;10:141-163.

17. Watters J, Biernacki P. Targeted sampling: options for the study of hidden populations. Soc Probl. 1989;36:416-430.

18. Boeri MW, Harbry L, Gibson D. A qualitative exploration of trajectories among suburban users of methamphetamine.JEthnogr Qual Res. 2009;3: $139-151$.

19. Boeri MW, Sterk CE, Elifson KW. Baby boomer drug users: career phases, social control and social learning theory. Sociol Inq. 2006;76(2): 264-291.

20. Sterk-Elifson C. Women and drug abuse: the application of qualitative research methods. In: Lambert E, Ashery R, Needle R, editors. Qualitative Methods in Drug Abuse and HIV Research. Washington DC: US Government Printing Office; 1995:66-83. NIDA Research Monograph 157; NIH publication; 95-4025.

21. Glaser BG, Strauss A. The Discovery of Grounded Theory: strategies for qualitative research. New York: Aldine; 1967.

22. Bruckner E, Mayer KU. Collecting life history data. In: Giele JZ, Elder GH Jr, editors. Methods of Life Course Research: Qualitative and Quantitative Approaches. Thousand Oaks (CA): Sage; 1998:169-199.

23. Murphy DA, Hser Y, Huang D, Brecht M, Herbeck D. Self report of longitudinal substance use: a comparison of the UCAL natural history interview and the addiction severity index. J Drug Issues. 2010;40(2):495-515.

24. Baltes BP, Brim OG Jr. Life Span Development and Behavior. New York: Academic Press; 1982.

25. Elder GH Jr. Life Course Dynamics. Ithaca (NY): Cornell University Press; 1985.

26. Laub JH, Sampson RJ. Turning points in the life course: why change matters in the study of crime. Criminology. 1993;31(3):301-326.

27. Scott JJ, Alwin D. Retrospective versus prospective measurement of life histories in longitudinal research. In: Giele JZ, Elder GH Jr. Methods of Life Course Research: Qualitative and Quantitative Approaches. Thousand Oaks (CA): Sage; 1998:98-127.

28. Agar M. The Professional Stranger: An Informal Introduction to Ethnography. New York: Academic Press; 1980. 
29. Becker HS. Tricks of the Trade: How to Think about Your Research While You're Doing It. Chicago: University of Chicago Press; 1998.

30. Darke S. Self-report among injecting drug users: a review. Drug Alcohol Depend. 1998;51(3):253-263.

31. Lambert E, editor. The Collection and Interpretation of Data from Hidden Populations. Washington DC: US Government Printing Office; 1990. NIDA Research Monograph 98; NIH publication; 90-1678.

32. Shaw VN. Research with participants in problem experience: challenges and strategies. Qual Health Res. 2005;15(6):841-854.

33. Fendrich M, Mackesy-Amiti ME, Wislar JS, Goldstein P. The Reliability and Consistency of Drug Reporting in Ethnographic Samples. Washington DC: US Government Printing Office; 1997:81-107.

34. Fontana A, Frey JH. Interviewing: the art of science. In: Denzin NK, Lincoln YS. Collecting and Interpreting Qualitative Materials. Thousand Oaks (CA): Sage; 1998:47-78.

35. Sobell LC, Sobell MB, Leo GI, Cancilla A. Reliability of a timeline method: assessing normal drinkers' reports of recent drinking and a comparative evaluation across several populations. Br J Addict. 1988;83(4): 393-402.

36. Guest G, BunceA, Johnson L. How many interviews are enough? An experiment with data saturation and variability. Field Methods. 2006;18(1): 59-82.

37. Johnson PB, Richter L. Research note: what if we're wrong? Some possible implications of systematic distortions in adolescents' self-reports of sensitive behaviors. J Drug Issues. 2004;34(4):951-970.
38. Teddlie C, Yu F. Mixed methods sampling: a typology with examples. J Mix Method Res. 2007;1(1):77-100.

39. Ragin C. Redesigning Social Inquiry: Fuzzy Sets and Beyond. Chicago: University of Chicago Press; 2008.

40. Kaplan CD, Lambert EY. The daily life of heroin-addicted persons: the biography of specific methodology. In: Lambert EY, Ashery RS, Needle RH, editors. Qualitative Methods in Drug Abuse and HIV Research. Washington DC: US Government Printing Office; 1995. NIDA Research Monograph 157; NIH Publication; 95-4025.

41. Wiebel WW. Identifying and gaining access to hidden populations. In: Lambert E, editor. NIDA Research Monograph 98. Washington DC: US Government Printing Office; 1990:4-11. NIH Publication; 90-1678.

42. Grannis R. Paths and semipaths: reconceptualizing structural cohesion in terms of directed relations. Sociol Methodol. 2009;39:117-150.

43. Degenhardt L, Mathers B, Vickerman P, Rhodes T, Latkin C, Hickman M. Prevention of HIV infection for people who inject drugs: why individual, structural, and combination approaches are needed. Lancet. 2010;376(9737):285-301.

44. Strathdee S, Hallett TB, Bobrova N, et al. HIV and risk environment for injecting drug users: the past, present, and future. Lancet. 2010; 376(9737):368-384.

45. Benshoff JJ, Harrawood L, Koch DS. Substance abuse and the elderly: unique issues and concerns. J Rehabil. 2003;69(2):43-48.

46. Lyons T, Lurigio AJ. The role of recovery capital in the community reentry of prisoners with substance use disorders. J Offender Rehabil. 2010;49:1-11.
Substance Abuse and Rehabilitation

\section{Publish your work in this journal}

Substance Abuse and Rehabilitation is an international, peer-reviewed, open access journal publishing original research, case reports, editorials, reviews and commentaries on all areas of addiction and substance abuse and options for treatment and rehabilitation. The manuscript management system is completely online and includes a very quick and

\section{Dovepress}

fair peer-review system. Visit http://www.dovepress.com/testimonials. php to read real quotes from published authors. 